\title{
Nuevos registros de Lagostomus Brookes, 1828 (Rodentia, Chinchillidae) en el Mioceno tardío de Argentina y su importancia bioestratigráfica
}

\author{
Luciano Luis Rasia ${ }^{1}$, Ricardo A. Bonini ${ }^{2}$, Adriana M. Candela ${ }^{1}$ \\ ' CONICET, División Paleontología Vertebrados, Museo de La Plata, Paseo del Bosque s/n, B1900FWA, La Plata, Argentina. \\ lucianorasia@conicet.gov.ar; acandela@fcnym.unlp.edu.ar \\ 2 INCUAPA-CONICET, Facultad de Ciencias Sociales, Universidad Nacional del Centro, Del Valle 5737, B7400, Olavarría, Argentina. \\ rbonini@fcnym.unlp.edu.ar
}

\begin{abstract}
RESUMEN. En este trabajo se dan a conocer dos nuevos registros de Lagostomus del Mioceno tardío de Argentina. Una mandíbula derecha proveniente de la Formación Huachipampa (Loma del Camello, provincia de San Juan) es asignada a Lagostomus telenkechanum, previamente registrada en la Formación Arroyo Chasicó (piso/edad Chasiquense, Mioceno tardío; Arroyo Chasicó, provincia de Buenos Aires). Un fragmento de cráneo procedente de la Formación Cerro Azul (Salinas Grandes de Hidalgo, provincia de La Pampa) es aquí referido a L. pretrichodactyla, antes registrado en varias unidades asignadas al piso/edad Huayqueriense (Mioceno tardío) en las provincias de Catamarca y Mendoza y en sedimentos de edad incierta en la provincia de Buenos Aires. La presencia de L. telenkechanum en la Formación Huachipampa indica una edad Chasiquense para esta unidad, lo que corrobora hipótesis previas, y extiende el área de distribución de esta especie. La presencia de L. pretrichodactyla en la Formación Cerro Azul sugiere una edad miocena tardía (probablemente Mesiniana) para esta unidad, al menos en Salinas Grandes de Hidalgo, coincidente con los resultados de estudios previos. Estos nuevos registros permiten reconocer el valor bioestratigráfico de las especies de Lagostomus a una escala geográfica mayor a la previamente considerada.
\end{abstract}

Palabras clave: Rodentia, Caviomorpha, Mioceno, Argentina, Bioestratigrafia.

\begin{abstract}
New records of Lagostomus Brookes (Rodentia, Chinchillidae) in the late Miocene of Argentina and its biostratigraphic importance. In this work, we present two new records of Lagostomus from the late Miocene of Argentina. A right mandible from the Huachipampa Formation (Loma del Camello, San Juan Province) is assigned to Lagostomus telenkechanum, previously recorded in the Arroyo Chasicó Formation (Chasicoan Stage/Age, late Miocene; Arroyo Chasicó, Buenos Aires Province). A skull fragment from the Cerro Azul Formation (Salinas Grandes de Hidalgo, La Pampa Province) is referred to L. pretrichodactyla, previously recorded in several units assigned to the Huayquerian Stage/Age (late Miocene) in Catamarca and Mendoza provinces, and sediments of uncertain age in Buenos Aires Province. The presence of L. telenkechanum in the Huachipampa Formation supports a Chasicoan age for this unit, which corroborates previous hypotheses, and extends the area of distribution of this species. The presence of L. pretrichodactyla in the Cerro Azul Formation suggests a late Miocene age (probably Messinian) for this unit, at least at Salinas Grandes de Hidalgo, supporting previous hypothesis. These new records allow recognizing the biostratigraphic value of Lagostomus to a higher geographic scale than previously considered.
\end{abstract}




\section{Introducción}

La familia Chinchillidae es un grupo de roedores caviomorfos poco diverso en la actualidad en comparación con otros clados (e.g., Octodontidae, Echimyidae, Caviidae), con seis especies incluidas en tres géneros (Nowak, 1991; Spotorno y Patton, 2015; Spotorno y Valladares, 2016). Chinchilla y Lagidium, con dos y tres especies respectivamente, son agrupados dentro de Chinchillinae. Lagostomus, con la única especie viviente L. maximus (Desmarest, 1817), conocida vulgarmente como vizcacha, se incluye en Lagostominae (e.g., Rowlands, 1974; Jackson et al., 1996; Spotorno et al., 2004; Spotorno y Patton, 2015; Spotorno y Valladares, 2016; Valladares et al., 2018).

La vizcacha se halla distribuida en Argentina, Bolivia y Paraguay y ocupa una gran variedad de ambientes, desde pastizales subtropicales hasta matorrales semidesérticos en las regiones Pampeana, del Monte y Chaqueña (e.g., Jackson et al., 1996; Spotorno y Patton, 2015). Son animales nocturnos, gregarios, con un marcado dimorfismo sexual, y habitan en complejos sistemas de cuevas que construyen cavando con sus miembros anteriores (e.g., Llanos y Crespo, 1952; Weir, 1974; Jackson et al., 1996).

Tradicionalmente se estimó que las vizcachas fósiles del piso/edad Chapadmalalense (Plioceno tardío) y las más antiguas que estas (Mioceno tardío-Plioceno temprano) pertenecían al género Lagostomopsis, originalmente propuesto por Kraglievich (1926) como un subgénero de Lagostomus y posteriormente utilizado como un género independiente (Kraglievich, 1934). Por otra parte, las especies poschapadmalalenses de vizcachas eran clasificadas como Lagostomus (e.g., Kraglievich, 1926, 1934; Pascual, 1966). Dada la similitud entre Lagostomus y Lagostomopsis (ver Francis y Mones, 1965), en trabajos recientes (e.g., Rasia, 2016; Rasia y Candela, 2017a, b) Lagostomopsis no es considerado un taxón válido y todas las especies extintas previamente comprendidas en este taxón son ahora incluidas en el género Lagostomus.

El registro fósil de Lagostomus es muy abundante en el sur de América del Sur, con restos encontrados en Argentina, Bolivia, Uruguay y sur de Brasil (e.g., Ameghino, 1889; Francis y Mones, 1968; Marshall y Sempere, 1991; Kerber et al., 2011; Rasia y Candela, 2013, 2017a, b; Ubilla y Rinderknecht, 2016). El reconocimiento más antiguo del género corresponde a Lagostomus telenkechanum Rasia y Candela, 2017, de la Formación Arroyo Chasicó (Mioceno tardío, piso/ edad Chasiquense), en la provincia de Buenos Aires (Rasia y Candela, 2017a). En el Mioceno tardío de las provincias de Catamarca y Mendoza se reconoce a L. pretrichodactyla (Rovereto, 1914) (e.g., Rovereto, 1914; Marshall y Patterson, 1981; Rasia, 2016) y en igual época en la provincia de Entre Ríos se encuentran L. antiquus Ameghino, 1883 y L. laminosus Ameghino, 1891 (Ameghino, 1883, 1891a; Nasif et al., 2013). Muchos de los registros de Lagostomus del Mioceno tardío de América del Sur no han sido identificados a nivel específico (e.g., Brandoni et al., 2012; Contreras y Baraldo, 2011; Goin et al., 2000), lo que dificulta evaluar su posible utilización en correlación bioestratigráfica.

En este trabajo se dan a conocer dos nuevos registros de Lagostomus provenientes del Mioceno tardío de Argentina, se justifica su asignación taxonómica a nivel específico, se incluye una exhaustiva comparación con todas las especies fósiles reconocidas y con la especie viviente del género, y se discute su valor bioestratigráfico.

\section{Materiales y métodos}

Se estudiaron dos materiales inéditos (MLP-Pv 57-XII-23-6 y MLP-Pv 65-VII-29-72) provenientes de sedimentos del Mioceno tardío de Argentina y se los comparó con los holotipos y material referido de todas las especies reconocidas de Lagostomus (ver Apéndice 1). Se utilizaron dos medidas para comparar las proporciones de los dientes yugales, el diámetro mesio-distal y el diámetro labio-lingual (modificado de Rasia y Candela, 2017a, b).

Abreviaturas institucionales. CICYTTP: Centro de Investigaciones Científicas y Transferencia Tecnológica de Diamante, Entre Ríos, Argentina; MACN: Colección Paleontología Vertebrados (Pv), Colección Ameghino (A), y Mastozoología (Ma), Museo Argentino de Ciencias Naturales Bernardino Rivadavia, Buenos Aires, Argentina; MASP: Museo Antonio Serrano, Paraná, Entre Ríos, Argentina; MLP: Colección Paleontología Vertebrados (Pv) y Mastozoología (Mz), Museo de La Plata, Buenos Aires, Argentina.

\section{Contexto geológico}

\subsection{Formación Huachipampa}

La Formación Huachipampa aflora en las sierras de Mogna, al norte de la ciudad de San Juan, provincia 
de San Juan, Argentina (Fig. 1). Con una potencia de $407 \mathrm{~m}$, esta unidad litológica es predominantemente pelítica, aunque sus primeros $70 \mathrm{~m}$ son arenosos (e.g., Milana et al., 2003).

Se interpreta que el ambiente de depositación de los sedimentos que constituyen esta formación fue un abanico aluvial distal que dio lugar, gradualmente, a una planicie deprimida con ríos permanentes (entrelazados someros y explayados), con crecidas periódicas, desarrollada en un clima árido, y a una capa freática alta que favoreció una vegetación densa (Johnson et al., 1986; Milana et al., 2003).
La edad de la Formación Huachipampa sería Tortoniana (ver Fig. 2), entre los 10,3 y los 8,4 Ma (Johnson et al., 1986) o entre los 9,2 y los $8 \mathrm{Ma}$ (Milana et al., 2003), y fue correlacionada con el piso/edad Chasiquense sobre la base de su fauna de vertebrados (Pascual y Bondesio, 1963; Bondesio et al., 1980).

\subsection{Formación Cerro Azul}

La Formación Cerro Azul se compone principalmente de limolitas y areniscas con intercalaciones de arcilitas hacia su base y calcretas

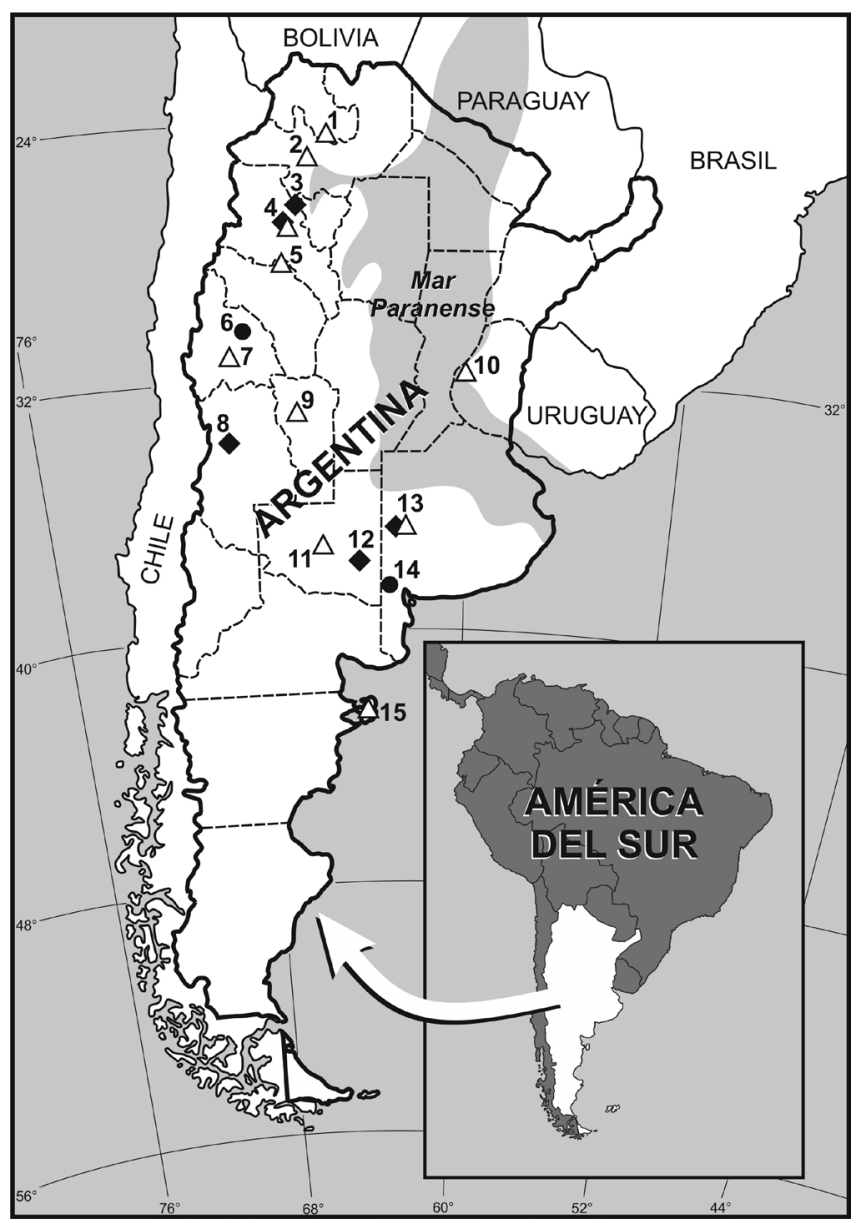

FIG. 1. Mapa de Argentina con la ubicación geográfica de las localidades mencionadas en el texto. 1. Los Alisos, Jujuy; 2. Valle de Tonco, Salta; 3. Valle de Santa María, Catamarca; 4. Corral Quemado, Catamarca; 5. El Degolladito, La Rioja; 6. Sierra de Mogna, San Juan; 7. Loma de las Tapias, San Juan; 8. Huayquerías de San Carlos, Mendoza; 9. Cantera Díaz Nogarol, San Luis; 10. Paraná, Entre Ríos; 11. Cerro La Bota, La Pampa; 12. Salinas Grandes de Hidalgo, La Pampa; 13. Lagunas Encadenadas del Oeste, Buenos Aires; 14. Arroyo Chasicó, Buenos Aires; 15. Península Valdés, Chubut; círculo negro: Lagostomus telenkechanum; rombo negro: L. pretrichodactyla; triángulo blanco: Lagostomus. Distribución del mar Paranense, transgresión marina del Mioceno tardío, sombreado en gris claro (según Aceñolaza, 2000). 


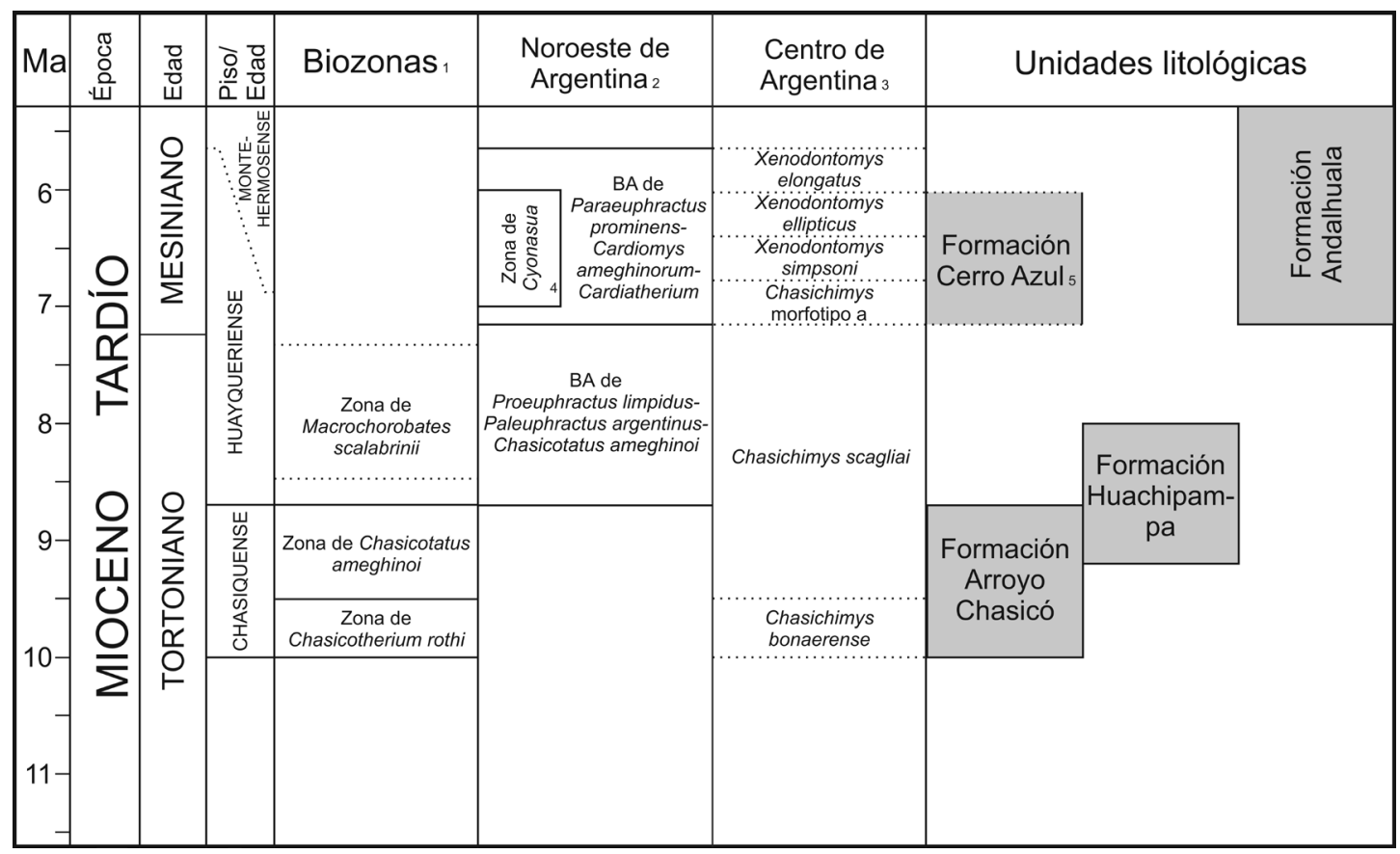

FIG. 2. Cuadro cronoestratigráfico de América del Sur. 1. Cione y Tonni, 2001, 2005; 2. Esteban et al., 2014; 3. Deschamps, 2005; Verzi et al., 2004, 2008; 4. Reguero y Candela, 2011; 5. Formación Cerro Azul en Salinas Grandes de Hidalgo. Las líneas punteadas indican límites sin edades absolutas.

hacia el tope de la sucesión; aflora mayormente en la provincia de La Pampa (e.g., Visconti et al., 2010).

Los sedimentos limosos habrían sufrido transporte eólico en suspensión y se habrían depositado durante períodos de lluvias. En épocas de menor tasa de sedimentación se formaron paleosuelos y en sectores específicos se desarrollaron lagos someros y cursos fluviales (Visconti et al., 2010).

Gran parte de esta unidad ha sido referida al piso/edad Huayqueriense (Mioceno tardío) sobre la base de su fauna de vertebrados (e.g., Esteban et al., 2001; Goin et al., 2000; Verzi et al., 2004, 2008). En la localidad de Salinas Grandes de Hidalgo, provincia de La Pampa (Fig. 1), la "formación" Epecuén ha sido considerada como sinónimo de la Formación Cerro Azul (e.g., Goin et al., 2000).

\section{El registro fósil de Lagostomus en el Mioceno tardío de América del Sur}

\subsection{Noroeste de Argentina}

Rovereto (1914) describió tres especies de vizcachas fósiles del "Araucanense" del valle de
Santa María, provincia de Catamarca ("Viscaccia" pretrichodactyla, "Viscaccia angulata" y "Viscaccia insolita"), cuyos materiales carecen de procedencia estratigráfica precisa. Posteriormente, Marshall y Patterson (1981) consideraron a "Viscaccia angulata" y "Viscaccia insolita" como sinónimos de Lagostomus pretrichodactyla, y reportaron además nuevos registros del Terciario tardío del Valle de Santa María y de la Puerta de Corral Quemado en la provincia de Catamarca (Fig. 1). Dos especímenes colectados en este mismo valle provienen del nivel XVIIIa y del nivel XX del "Araucanense" (perfil estratigráfico de Stahlecker en Marshall y Patterson, 1981), y corresponderían a la Formación Andalhuala (Bonini, 2014; Esteban et al., 2014). Entre estos dos niveles (Nivel XIX) se estima una edad cercana a los 6,02 Ma (ver Marshall y Patterson, 1981). Más recientemente, restos de Lagostomus sp. fueron encontrados en la provincia de Catamarca, en el tercio inferior de la Formación Andalhuala en la Puerta de Corral Quemado (Esteban et al., 2014), y en el Miembro El Jarillal de la Formación Chiquimil en Villavil (Herbst et al., 2000; Fig. 1). 
En la Formación Salicas, Mioceno tardío de la provincia de La Rioja (Fig. 1), se encontraron restos de Lagostomus cf. L. pretrichodactyla (Tauber, 2005) y Lagostomus sp. (Brandoni et al., 2012).

En la Formación Palo Pintado (Mioceno tardío, Chasiquense-Huayqueriense), en el Valle de Tonco, provincia de Salta (Fig. 1), se evidenciaron restos de Lagostomus sp. y Prolagostomus sp. (Zimicz et al., 2018). El material referido a Prolagostomus por Zimicz et al. (2018), un fragmento izquierdo de maxilar con P4-M3, carece de los rasgos característicos de este género (e.g., molares con hipoflexos sinuosos, tercer lofo del M3 laminar y orientado distal o distolingualmente; ver Rasia y Candela, 2019) por lo que es aquí considerado como Lagostomus sp.

En la Formación Guanaco (Mioceno tardío) en Los Alisos, provincia de Jujuy (Fig. 1), se registró Lagostomus sp. (Ercoli et al., 2019).

\subsection{Cuyo, Argentina}

Lagostomus pretrichodactyla fue reportada en el "Araucanense" de Huayquerías de San Carlos, provincia de Mendoza (Rovereto, 1914; ver Fig. 1) y posteriormente en la Formación Huayquerías (Mioceno tardío), en la misma localidad (Forasiepi et al., 2014, 2016; Bonini et al., 2016).

Restos de Lagostomus sp. fueron reportados para la Asociación A (de edad Chasiquense) y la Asociación B (de edad Huayqueriense) de la Formación Loma de las Tapias, en la provincia de San Juan (Contreras y Baraldo, 2011; ver Fig. 1).

\subsection{Sierras Pampeanas, Argentina}

Prado et al. (1998) informaron la presencia de restos de Lagostomus sp. para la Formación Río Quinto (Cantera Díaz Nogarol, provincia de San Luis; ver Fig. 1), del Mioceno tardío-Plioceno (ver Lucero, 2016).

\subsection{Región Pampeana, Argentina}

Lagostomus telenkechanum fue descrita para la Formación Arroyo Chasicó (Arroyo Chasicó, provincia de Buenos Aires; ver Fig. 1), de edad Chasiquense (Rasia y Candela, 2017a). Mientras que tradicionalmente se reconocen dos miembros de distinta edad para esta unidad (Miembro Vivero de edad Chasiquense Inferior y Miembro Las Barrancas de edad Chasiquense Superior; e.g., Cione et al., 2000; Cione y Tonni, 2001), estudios recientes sostienen que no hay evidencias litofaciales (Zárate et al., 2007) ni bioestratigráficas (Tomassini et al., 2017) para dividir la Formación Arroyo Chasicó en los dos miembros antes mencionados. La edad de la Formación Arroyo Chasicó se estima entre 10 y 8,7 Ma (Cione et al., 2000; Zárate et al., 2007).

En niveles Chasiquenses (Mioceno tardío) de la Formación Cerro Azul en el Cerro La Bota, provincia de La Pampa, se encontraron restos de Lagostomus sp. (Montalvo et al., 2019), al igual que en Salinas Grandes de Hidalgo, provincia de La Pampa (Goin et al., 2000).

En el "Irenense" del río Quequén Salado (provincia de Buenos Aires), se reconocen al menos dos asociaciones faunísticas (ver Pardiñas et al., 2017): una de edad Huayqueriense y otra más moderna de edad Montehermosense. En la Asociación Huayqueriense se reportó la presencia de Lagostomus euplasius (Ameghino, 1908) (ver Pardiñas et al., 2017), mientras que en la Asociación Montehermosense, L. incisus Ameghino, 1888 (ver Rasia y Candela, 2013; Rasia, 2016; Pardiñas et al., 2017). No existen dataciones para el "Irenense", y como remarcaron Pardiñas et al. (2017) no puede definirse con certeza si la Asociación Montehermosense es Mioceno tardía o Plioceno temprana.

En las Lagunas Encadenadas del Oeste (Fig. 1), en la provincia de Buenos Aires, se registraron Lagostomus pretrichodactyla y L. incisus, en sedimentos de edad incierta, pero que podrían haberse depositado entre el piso/edad Huayqueriense y el piso/edad Montehermosense (Bonini et al., 2017).

\subsection{Mesopotamia, Argentina}

Dos especies de Lagostomus, L. antiquus y L. laminosus (ver Ameghino, 1883, 1891a; Nasif et al., 2013) son reconocidas en el "Mesopotamiense" (="Conglomerado osífero", Mioceno tardío) de la Formación Ituzaingó, en la provincia de Entre Ríos (Fig. 1). Lagostomus pallidens, descrita por Ameghino (1886) para la misma unidad, es considerada nomen vanum (ver Nasif et al., 2013).

\subsection{Patagonia, Argentina}

En la Formación Puerto Madryn (Mioceno tardío, Piso/Edad Huayqueriense), en varias localidades de 
la península Valdés (Fig. 1), provincia del Chubut, se registró Lagostomus? (Dozo et al., 2002, 2010), lo que constituye el registro más austral del género.

\subsection{Bolivia}

Provenientes de los "Estratos Muyu Huasi” (Muyu Huasi, Bolivia), de supuesta edad Huayqueriense, se reportaron restos de Lagostomus sp. (Villarroel y Marshall, 1989; Marshall y Sempere, 1991).

\subsection{Uruguay}

Francis y Mones (1965) informaron la existencia de restos de "Lagostomus (Lagostomopsis) spicatus" para la "Formación Kiyú", de supuesta edad pliocena tardía, en el Departamento de San José, República Oriental del Uruguay. "Lagostomus spicatus" fue sinonimizada con Lagostomus incisus por Rasia y Candela (2013), quienes concluyeron también que el material descrito por Francis y Mones (1965) no corresponde a $L$. incisus, por lo que este quedó identificado como Lagostomus sp. Por otra parte, Sprechmann et al. (2000; ver también Perea et al., 2013) consideraron que la "Formación Kiyú" es en realidad parte de la Formación Camacho, de edad Huayqueriense, y la denominaron "litofacies Kiyú".

\section{Paleontología sistemática}

Orden Rodentia Bowdich, 1821

Suborden Hystricognathi Tullberg, 1899

Infraorden Caviomorpha Wood y Patterson

(en Wood, 1955)

Familia Chinchillidae Bennett, 1833

Subfamilia Lagostominae Wiegmann, 1835

Género Lagostomus Brookes, 1828

Especie tipo. Lagostomus trichodactylus Brookes, 1828 (=Dipus maximus Desmarest, 1817)

Lagostomus telenkechanum Rasia y Candela, 2017 Fig. 3A-D

Material referido. MLP 57-XII-23-6, mandíbula derecha incompleta con porción del incisivo y p4-m3 completos.

Procedencia geográfica y estratigráfica. El material fue colectado por el Dr. Héctor de la Mota en loma del Camello, sierra de Mogna, departamento de Jáchal, provincia de San Juan, Argentina (Fig. 1) y proviene de la Formación Huachipampa (Fig. 2), Mioceno tardío (piso/edad Chasiquense; Pascual y Bondesio, 1963; Bondesio et al., 1980).

Descripción comparativa. La porción preservada de la mandíbula es pequeña, similar en tamaño a los especímenes de Lagostomus telenkechanum de la Formación Arroyo Chasicó, a L. antiquus y a los individuos más pequeños de $L$. pretrichodactyla y L. euplasius. La muesca masetérica, sitio de inserción de la porción infraorbital del músculo masetero medio (sensu Woods y Howland, 1979), se encuentra entre el m1 y el m2, como en el material de Lagostomus telenkechanum proveniente de la Formación Arroyo Chasicó. La porción anterior de la cresta horizontal se encuentra a nivel del límite m2-m3, y está desconectada de la muesca masetérica, ubicándose dorso-posteriormente a esta última, igual que en otras especies de Lagostomus.

Las proporciones y los tamaños de los molariformes son comparables a los del material referido de Lagostomus telenkechanum proveniente de la Formación Arroyo Chasicó (ver Tabla 1 y Figs. 3C, 3D, 3F y 4), aunque son más comprimidos mesiodistalmente que los de L. pretrichodactyla, L. compressidens (Ameghino, 1908), L. debilis Ameghino, 1889, L. minimus Ameghino, 1889, L. heterogenidens Ameghino, 1889, L. cavifrons Ameghino, 1889, L. egenus Ameghino, 1891b y L. maximus, y menos que los de L. incisus; es decir, son similares a los de L. antiquus y L. euplasius.

La porción preservada del incisivo presenta el esmalte de color amarillento, como los especímenes de Lagostomus telenkechanum de la Formación Arroyo Chasicó. Este rasgo también se observa en Lagostomus euplasius, del Chapadmalalense de la provincia de Buenos Aires (Rasia y Candela, 2017b), aunque el tamaño de L. euplasius es considerablemente mayor (ver Fig. 4).

Comentarios. El material recuperado de la Formación Huachipampa es referido a Lagostomus telenkechanum por presentar un tamaño y una morfología de la mandíbula similares, y por el tamaño y proporciones de los molariformes, los cuales son más comprimidos mesio-distalmente que en L. maximus, L. cavifrons, L. egenus, L. debilis, L. minimus, L. heterogenidens, L. compressidens y $L$. euplasius, pero menos comprimidos que L. laminosus y L. incisus; asemejándose también a las proporciones de los molariformes de L. antiquus y L. euplasius. De esta última especie se diferencia 
A
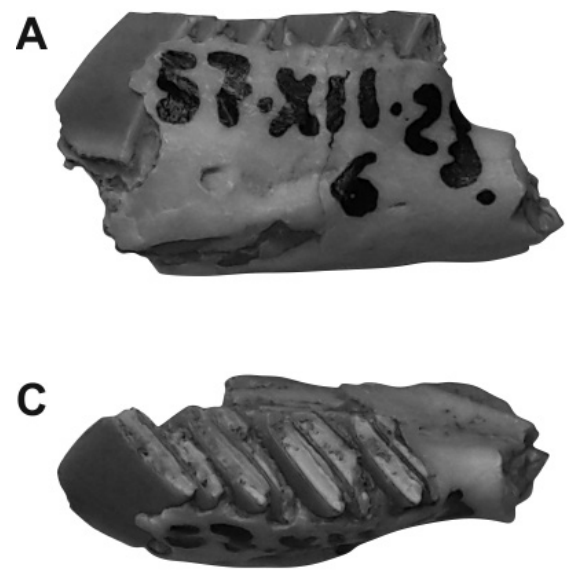

E

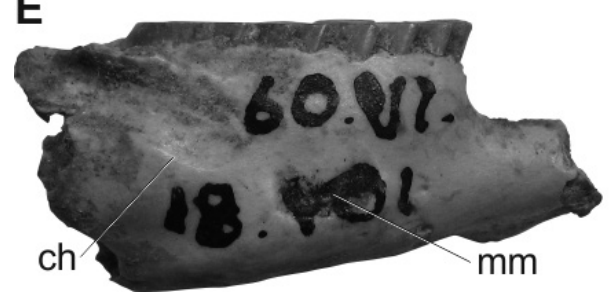

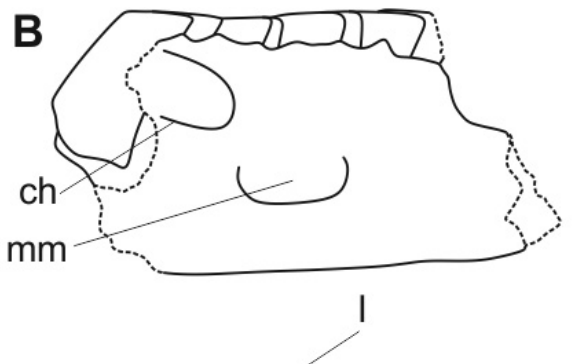
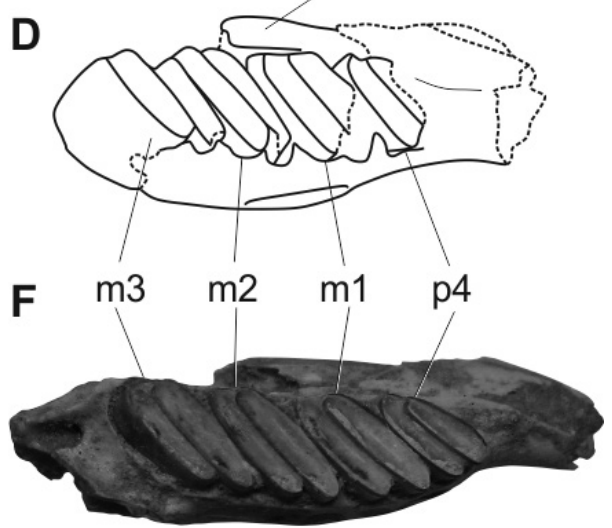

FIG. 3. Material de Lagostomus telenkechanum. A-D. MLP-Pv 57-XII-23-6, mandíbula derecha en vista lateral (A-B) y oclusal (C-D), proveniente de la Formación Huachipampa, San Juan. E-F. MLP-Pv 60-VI-18-101, mandíbula derecha en vista lateral (E) y oclusal (F), proveniente de la Formación Arroyo Chasicó, Buenos Aires. Abreviaturas: I: incisivo; m1: primer molar; m2: segundo molar; m3: tercer molar; mm: muesca masetérica; p4: cuarto premolar. Escala $1 \mathrm{~cm}$.

TABLA 1. MEDIDAS DENTARIAS (EN MILÍMETROS) DE LAGOSTOMUS TELENKECHANUM.

\begin{tabular}{llccccccccc}
\hline \multirow{2}{*}{ Material } & \multicolumn{1}{c}{$\begin{array}{c}\text { Procedencia } \\
\text { estratigráfica }\end{array}$} & MD & LL & MD & LL & MD & LL & MD & LL \\
\hline MLP-Pv 57-XII-23-6 & Fm. Huachipampa & - & 5,95 & 3,07 & 6,87 & 2,59 & 7,16 & 2,72 & 6,45 \\
MLP-Pv 60-VI-18-107 & Fm. Arroyo Chasicó & 2,3 & 4,47 & 2,32 & 5,09 & 2,29 & 4,98 & 2,24 & 4,63 \\
MLP-Pv 60-VI-18-101 & Fm. Arroyo Chasicó & 2,39 & 5,07 & 2,68 & 6,65 & 2,72 & 6,82 & 2,86 & 6,67 \\
MLP-Pv 76-VI-12-47 & Fm. Arroyo Chasicó & 2,76 & 5,32 & 2,99 & 6,78 & 2,75 & 6,91 & 2,75 & 6,2 \\
MLP-Pv 55-IV-28-42 & Fm. Arroyo Chasicó & 2,64 & 4,97 & 2,87 & 5,89 & 2,68 & 5,66 & - & - \\
MLP-Pv 55-IV-28-44 & Fm. Arroyo Chasicó & 2,21 & 4,74 & 2,65 & 5,62 & - & - & - & - \\
MLP-Pv 92-XI-19-1 & Fm. Arroyo Chasicó & 2,66 & 5,73 & - & - & - & - & - & - \\
\hline
\end{tabular}

LL: diámetro labio-lingual; MD: diámetro mesio-distal.

principalmente por el tamaño notablemente menor, mientras que de L. antiquus se diferencia por presentar los molariformes levemente más comprimidos mesio-distalmente y por tener el esmalte de los incisivos de color amarillento. Tanto el material de L. telenkechanum de la Formación Huachipampa como el de L. antiquus son bastante fragmentarios, por lo que no es posible una comparación más detallada. 


\section{Proporciones del $\mathrm{m} 1$ en Lagostomus}

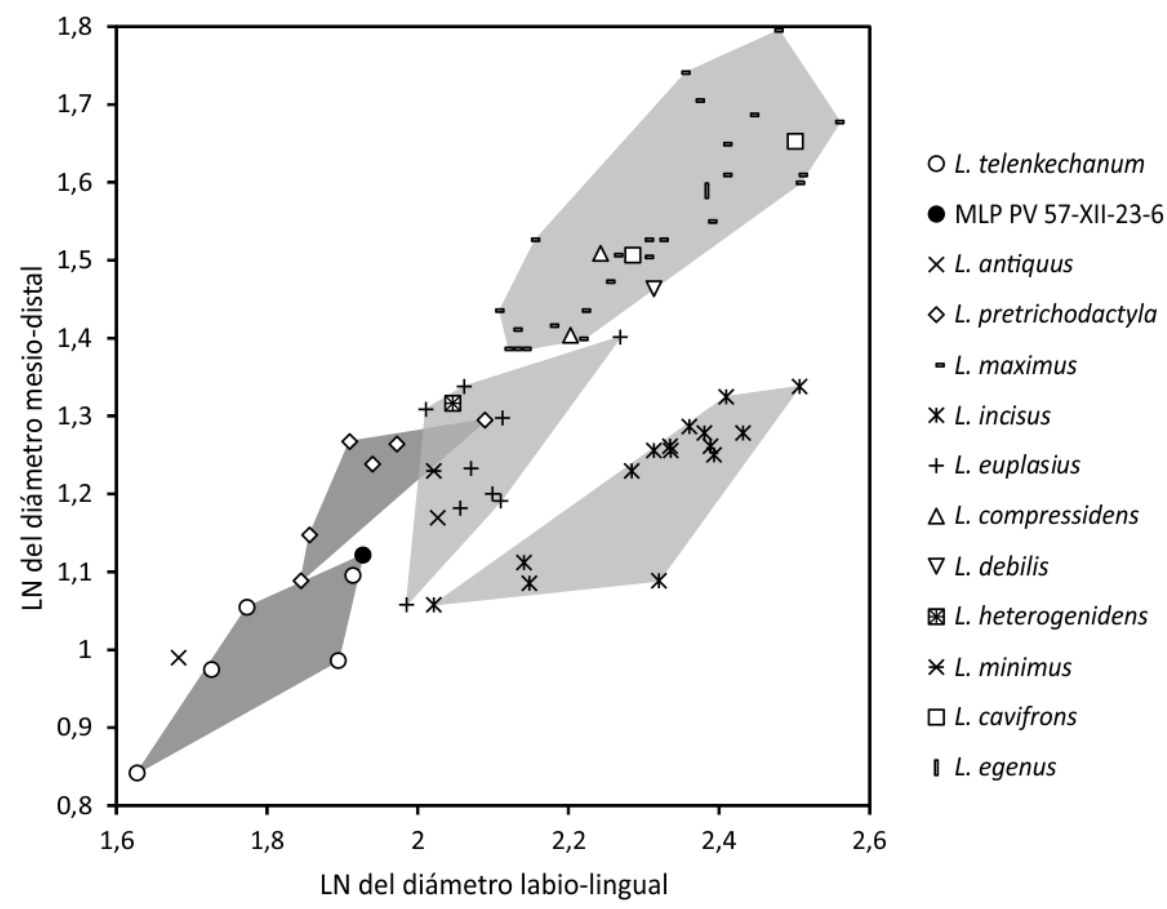

FIG. 4. Gráfico bivariado del logaritmo natural (LN) del diámetro labio-lingual y mesio-distal del primer molar inferior (m1) en especies de Lagostomus.

\section{Lagostomus pretrichodactyla (Rovereto, 1914) Fig. 5A-F}

Material referido. MLP 65-VII-29-72, porción anterior del cráneo, que incluye parte de los nasales, la parte anterior de los frontales, los premaxilares y maxilares, parte del arco cigomático derecho y los P4-M3 derechos e izquierdos.

Procedencia geográfica y estratigráfica. El material fue colectado por R. Pascual, E. J. Ortega Hinojosa y E. P. Tonni en Salinas Grandes de Hidalgo, provincia de La Pampa (Fig. 1). Proviene de la "formación" Epecuén, que en la localidad antes mencionada se considera sinónimo de la Formación Cerro Azul (Fig. 1), Mioceno tardío (piso/edad Huayqueriense; ver Goin et al., 2000; Fig. 2).

Descripción comparativa. La porción preservada del cráneo se asemeja en tamaño a individuos de Lagostomus euplasius. En la vista dorsal, los premaxilares sobrepasan posteriormente a los nasales (Fig. 5A-B), como en individuos de Lagostomus pretrichodactyla provenientes de Catamarca
(Fig. 5G), y en L. incisus y L. euplasius; estos se diferencian de $L$. compressidens, $L$. cavifrons y $L$. maximus. El foramen interpremaxilar es ancho y elongado anteroposteriormente (Fig. 5C-D), rasgo compartido por Lagostomus pretrichodactyla, L. euplasius, L. compressidens y por la mayoría de los individuos de L. maximus. El foramen incisivo es grande y ancho en su parte posterior (Fig. 5CD). Los procesos palatinos de los premaxilares son fuertes y posteriormente dirigidos, sobresaliendo ventralmente al plano del diastema, y diferenciándose de Lagostomus incisus. La pobre preservación del cráneo no permite una comparación más detallada.

Las proporciones de los molariformes superiores son semejantes a aquellas de los especímenes de Lagostomus pretrichodactyla reportados por Rovereto (1914) y Marshall y Patterson (1981) para la provincia de Catamarca (ver Tabla 2 y Figs. 5C, 5D, 5H y 6), aunque son menos comprimidos mesio-distalmente que los de L. telenkechanum, L. euplasius y L. incisus; se asemejan a los de $L$. compressidens, $L$. cavifrons y L. maximus. 
A

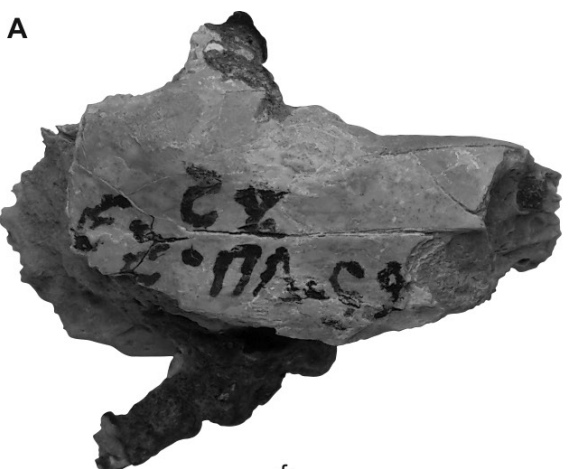

B
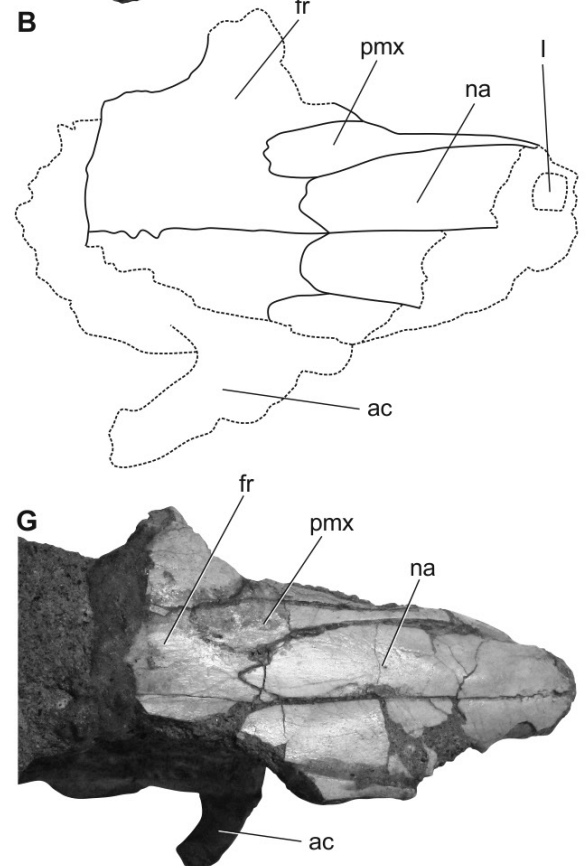

C

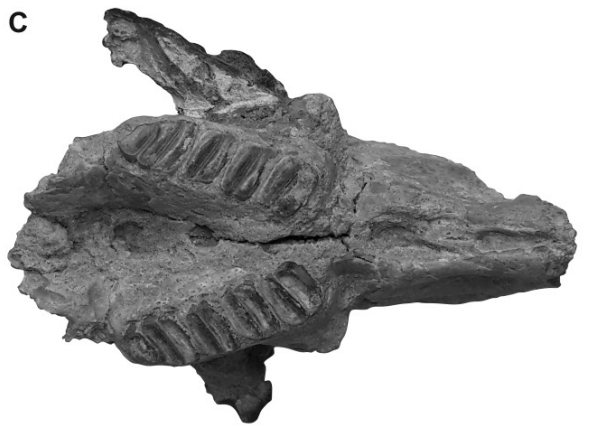

D

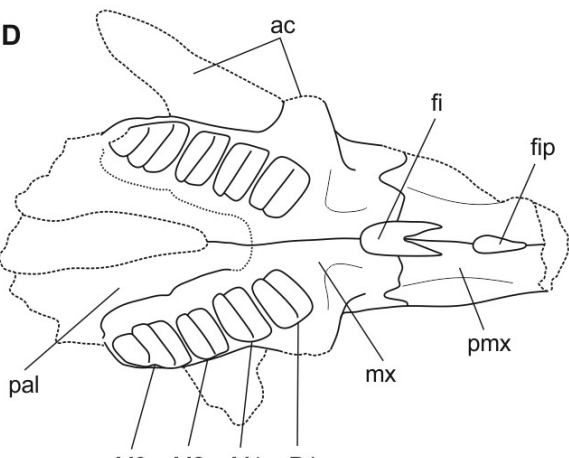

M3 $\quad$ M2 $\quad$ M1 $\quad$ P4

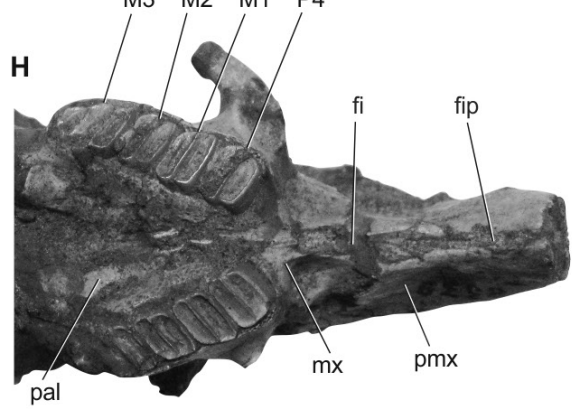

E
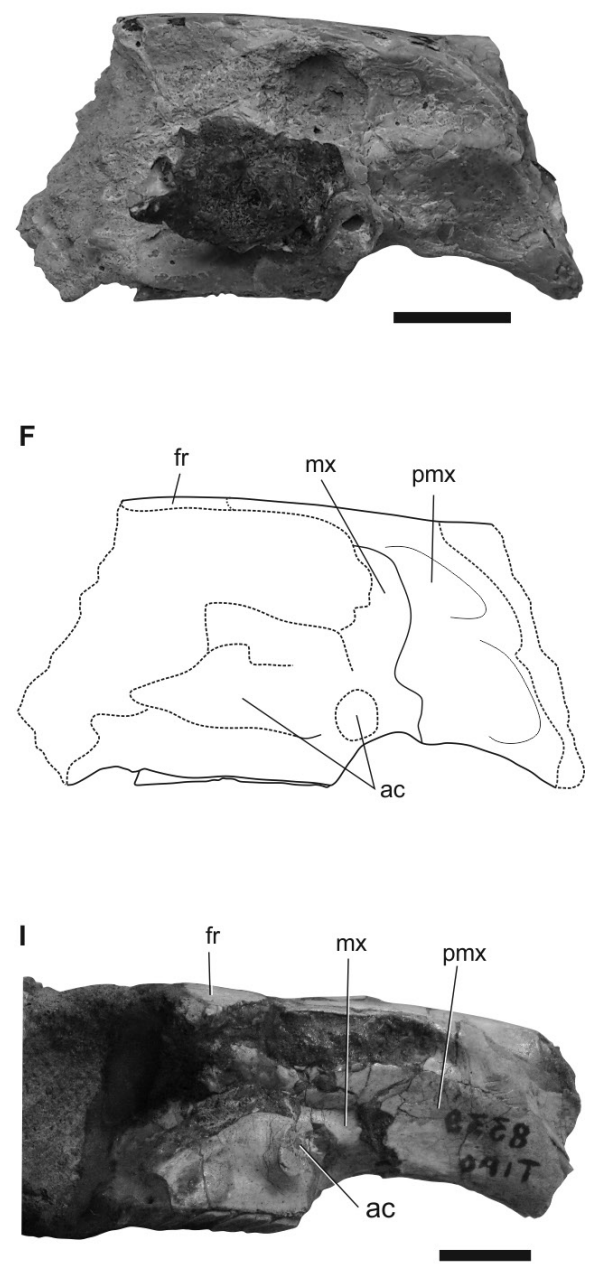

FIG. 5. Material de Lagostomus pretrichodactyla. A-F. MLP-Pv 65-VII-29-72, de la Formación Cerro Azul, La Pampa, en vista dorsal (A-B), ventral (C-D) y lateral (E-F). G-I. MACN-Pv 8339 (holotipo), del "Araucanense" de Catamarca, en vista dorsal (G), ventral (H) y lateral (I). Abreviaturas: ac: arco cigomático; fi: foramen incisivo; fip: foramen interpremaxilar; fr: rrontal; I: incisivo; M1: primer molar; M2: segundo molar; M3: tercer molar; mx: maxilar; na: nasal; pal: Palatino; P4: cuarto premolar; pmx: premaxilar. Escala $1 \mathrm{~cm}$. 
TABLA 2. MEDIDAS DENTARIAS (EN MILÍMETROS) DE LAGOSTOMUS PRETRICHODACTYLA.

\begin{tabular}{lccccccccccc}
\hline \multirow{2}{*}{ Material } & $\begin{array}{c}\text { Procedencia } \\
\text { estratigráfica }\end{array}$ & MD & LL & MD & LL & MD & LL & MD & LL \\
\hline MLP-Pv 65-VII-29-72 & Fm. Cerro Azul & 4,07 & 5,49 & 3,91 & 5,77 & 3,45 & 5,52 & 5,92 & 5,83 \\
MACN-Pv 8340 & "Araucanense" & 4,47 & 5,92 & 3,96 & 5,79 & 3,79 & 5,88 & 6,18 & 6,24 \\
MACN-Pv 8338 & "Araucanense" & 3,51 & 3,75 & 3,37 & 4,9 & 3,02 & 4,6 & - & - \\
MACN-Pv 8212 & "Araucanense" & 3,72 & 5,53 & 3,58 & 5,27 & 3,58 & 5,24 & 4,92 & 5,21 \\
MACN-Pv 8339 & "Araucanense" & 4,05 & 5,82 & 3,73 & 6,08 & 3,65 & 5,88 & 6,46 & 6,48 \\
MACN-Pv 8337 & "Araucanense" & 4,12 & 5,33 & 3,7 & 4,85 & 3,52 & 4,67 & 5,99 & 5,22 \\
MACN-Pv 8345 & "Araucanense" & 3,96 & 5,67 & 3,69 & 5,38 & 3,33 & 5,12 & - & 5,34 \\
\hline
\end{tabular}

LL: diámetro labio-lingual; MD: diámetro mesio-distal.

\section{Proporciones M1 en Lagostomus}

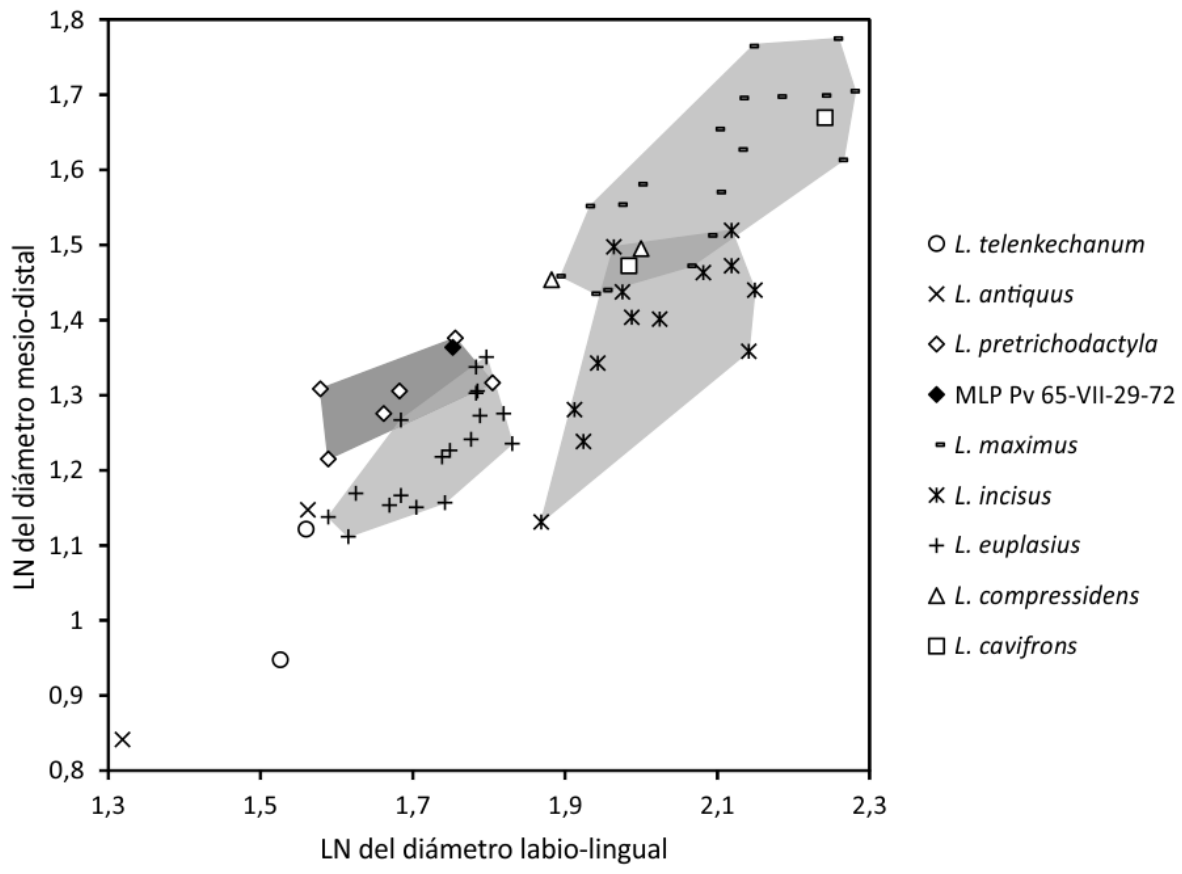

FIG. 6. Gráfico bivariado del logaritmo natural (LN) del diámetro labio-lingual y mesio-distal del primer molar superior (M1) en especies de Lagostomus.

Comentarios. El material proveniente de Salinas Grandes de Hidalgo es asignado a Lagostomus pretrichodactyla por compartir con esta especie el mismo tamaño y varios rasgos craneanos (e.g., premaxilares que sobrepasan posteriormente a los nasales, foramen interpremaxilar ancho y elongado anteroposteriormente, foramen incisivo grande y ancho en su parte posterior, procesos palatinos de los premaxilares fuertes y que sobresalen ventralmente al plano del diastema), por el tamaño y proporciones de los molariformes, los cuales son menos comprimidos mesio-distalmente que los de $L$. telenkechanum, $L$. euplasius y L. incisus, se asemejan a los de L. compressidens, $L$. cavifrons y L. maximus, y difiere de 
estas últimas tres especies por el tamaño y anatomía craneana. Si bien los rasgos craneanos no son exclusivos de L. pretrichodactyla, ya que también son compartidos con L. euplasius, el material se diferencia de esta última especie por poseer los molariformes menos comprimidos mesio-distalmente. Cabe destacar que el material aquí estudiado corresponde a un individuo subadulto/adulto, dados varios rasgos craneanos (e.g., proporción nasales/frontales, tamaño del foramen incisivo respecto del diastema; ver Rasia, 2016).

\section{Discusión y conclusiones}

La presencia de Lagostomus telenkechanum en sedimentos referidos al piso/edad Chasiquense, de edad Tortoniana (i.e., Formación Arroyo Chasicó y Formación Huachipampa), en dos localidades separadas geográficamente (en la región Pampeana y Cuyo), indica que esta especie estuvo ampliamente distribuida durante el Chasiquense, lo que le daría un alto potencial como herramienta bioestratigráfica. Además, este es el primer registro de L. telenkechanum fuera de su localidad tipo (Arroyo Chasicó; Rasia y Candela, 2017a), ampliando así su área de distribución.

Lagostomus pretrichodactyla es registrada en varias unidades referidas al piso/edad Huayqueriense (i.e., Formación Andalhuala, Formación Huayquerías, Formación Cerro Azul). La presencia de ciertos roedores octodontoideos como Phtoramys hidalguense, Reigechimys octodontiformis y Pampamys emmonsae en niveles de la Formación Cerro Azul aflorantes en Salinas Grandes de Hidalgo (ver Goin et al., 2000) indican una edad Huayqueriense Tardía (Mioceno tardío; ver Verzi et al., 2008) para esta unidad (al menos en esa localidad). El registro de Lagostomus pretrichodactyla en la Formación Cerro Azul apoya la asignación a una edad miocena tardía para esta unidad, al menos en Salinas Grandes de Hidalgo. Dado que el límite entre el piso/edad Huayqueriense y el piso/ edad Montehermosense no está definido y distintos autores lo ubican en el Mioceno tardío (e.g., Cione y Tonni, 1995, 2001; Reguero y Candela, 2011) o en el límite Mioceno tardío/Plioceno temprano (e.g., Tomassini et al., 2013), no sería conveniente referir taxativamente la Formación Cerro Azul en Salinas Grandes de Hidalgo al Huayqueriense, sino al Mioceno más tardío, probablemente al Mesiniano. Además, la presencia de L. pretrichodactyla se registra en varias localidades separadas geográficamente (en la región Pampeana, Cuyo y Noroeste) referidas al Mioceno tardío, por lo que también tendría un alto potencial como herramienta bioestratigráfica.

La identificación de la misma especie de Lagostomus en localidades geográficamente alejadas unas de otras (región Pampeana y Cuyo para L. telenkechanum, y región Pampeana, Cuyo y NO de Argentina para L. pretrichodactyla) y acotadas temporalmente (edad Tortoniana, piso/edad Chasiquense para Lagostomus telenkechanum y edad Mesiniana, Mioceno tardío para L. pretrichodactyla) permite reconocer que las especies más antiguas de Lagostomus tienen importancia bioestratigráfica a escala regional.

A escala más local, dos especies de Lagostomus, Lagostomus incisus (ver Rasia y Candela, 2013, 2017b; Rasia, 2016) y L. euplasius (ver Rasia, 2016; Rasia y Candela, 2017b), registradas en sedimentos pliocenos (pisos/edades Montehermosense-Chapadmalalense) que afloran en varias localidades de la provincia de Buenos Aires, fueron reconocidas por su utilidad bioestratigráfica.

Por lo expuesto anteriormente, las especies de Lagostomus del Mioceno tardío y Plioceno de la Argentina tienen valor como herramientas bioestratigráficas tanto en el ámbito local como a mayor escala geográfica. Además, dado el abundante registro fósil de Lagostomus en el Mioceno tardío del sur de América del Sur, el estudio sistemático y anatómico más profundo de aquellos registros identificados solo a nivel de género podría contribuir de manera sustancial a una mejor comprensión de la bioestratigrafía Sudamericana.

Durante el Mioceno tardío la distribución geográfica del género Lagostomus fue más amplia que en la actualidad, ya que los registros de Península Valdés (provincia del Chubut), departamento de San José (Uruguay) y Muyu Huasi (Bolivia) exceden la distribución de la especie viviente, Lagostomus maximus. Por otra parte, la gran mayoría de los registros de Lagostomus durante el Mioceno tardío se encuentran al oeste de territorio ocupado por el mar Paranense (Fig. 1), excepto los registros de la provincia de Entre Ríos y en Uruguay, que se encuentran al este de dicha transgresión marina. Por lo tanto, la diferenciación del género Lagostomus y su posterior expansión geográfica debió ocurrir en el Mioceno tardío más bajo (probablemente durante el Chasiquense), previamente a la transgresión Paranense, datada entre 8,85 y 6 Ma en Entre Ríos, Buenos Aires y Uruguay (Del Río et al., 2018). 


\section{Agradecimientos}

Agradecemos a M. Reguero (MLP), A. Scarano (MLP), A. Kramarz (MACN), L. Chornogubsky (MACN), M. Ezcurra (MACN), P. Teta (MACN) y S. Lucero (MACN) por facilitar el acceso a las colecciones a su cuidado. Agradecemos a L. Kerber y a un revisor anónimo por la lectura crítica del manuscrito.

\section{Referencias}

Aceñolaza, F.G. 2000. La Formación Paraná (Mioceno medio): estratigrafía, distribución regional y unidades equivalentes. In El Neógeno de Argentina (Aceñolaza, F.G.; Herbst, R.; editores). Instituto Superior de Correlación Geológica (INSUGEO), Serie Correlación Geológica 14: 9-28.

Ameghino, F. 1883. Sobre una nueva colección de mamíferos fósiles recogidos por el Profesor Scalabrini en las barrancas del Paraná. Boletín de la Academia Nacional de Ciencias de Córdoba 5: 112-113.

Ameghino, F. 1886. Contribución al conocimiento de los mamíferos fósiles de los terrenos terciarios antiguos del Paraná. Boletín de la Academia Nacional de Ciencias de Córdoba 9: 5-228.

Ameghino, F. 1888. Lista de las especies de mamíferos fósiles del Mioceno superior de Monte Hermoso, hasta ahora conocidas. Editorial Coni: 21 p. Buenos Aires.

Ameghino, F. 1889. Contribución al conocimiento de los mamíferos fósiles de la República Argentina. Boletín de la Academia Nacional de Ciencias de Córdoba 6: 1-127.

Ameghino, F. 1891a. Mamíferos y aves fósiles argentinos. Especies nuevas, adiciónes y correcciónes [sic.]. Revista Argentina de Historia Natural 1: 240-259.

Ameghino, F. 1891b. Caracteres diagnósticos de cincuenta especies nuevas de mamíferos fósiles argentinos. Revista Argentina de Historia Natural 1: 129-167.

Ameghino, F. 1908. Las Formaciones sedimentarias de la región litoral de Mar del Plata y Chapadmalal. Anales del Museo Nacional de Buenos Aires 10: 342-428.

Bennett, E.T. 1833. Remarks on the family Chinchillidae and on a new genus referrible [sic.] to it. Proceedings of the Zoological Society of London 1: 57-60.

Bondesio, P.; Laza, J.H.; Scillato Yané, G.J.; Tonni, E.P.; Vucetich, M.G. 1980. Estado actual del conocimiento de los vertebrados de la Fm. Arroyo Chasicó (Plioceno temprano) de la Provincia de Buenos Aires. In Congreso Argentino de Paleontología y Bioestratigrafía, No. 2 y Congreso Latinoamericano de Paleontología, No. 1, Actas 3: 101-127. Buenos Aires.
Bonini, R.A. 2014. Bioestratigrafía y diversidad de los mamíferos del Neógeno de San Fernando y Puerta de Corral Quemado (Catamarca, Argentina). Tesis Doctoral (Inédito), Universidad Nacional de La Plata, Facultad de Ciencias Naturales y Museo: 337 p.

Bonini, R.A.; Forasiepi, A.M.; Prevosti, F.J.; Garrido, A.C.; Barbeau, D.L.; Turazzini, G.F.; Echarri, S.; Pujos, F.; MacPhee, R.; Verzi, D.; Vera, B.; Cerdeño, E.; Pérez, M.E.; Rasia, L.L.; Esteban, G.; De La Fuente, M.S. 2016. Avances en paleontología, estratigrafía y edad de la Formación Huayquerías (Mioceno tardío, Mendoza). Ameghiniana 53 (6): 50-51R.

Bonini, R.A.; Scanferla, A.C.; Candela, A.M.; Rasia, L.L.; Schmidt, G.I. 2017. Estudio preliminar de una nueva fauna de mamíferos del Neógeno de las Lagunas Encadenadas del Oeste de la Provincia de Buenos Aires. In Congreso Geológico Argentino, No. 20 y Simposio del Mioceno-Pleistoceno del Centro y Norte de Argentina, No. 4, Actas: 19-24. San Miguel de Tucumán.

Bowdich, T.E. 1821. An analysis of the natural classifications of Mammalia for the use of students and travelers. Smith: 115 p. París.

Brandoni, D.; Schmidt, G.I.; Candela, A.M.; Noriega, J.I.; Brunetto, E.; Fiorelli, L.E. 2012. Mammals from the Salicas Formation (Late Miocene), La Rioja Province, Northwestern Argentina: Palobiogeography, Age, and Paleoenvirnment. Ameghiniana 49 (3): 375-387.

Brookes, J. 1828. A new genus of the order Rodentia. Transactions of the Linnean Society 16: 96-105.

Cione, A.L.; Tonni, E.P. 1995. Los estratotipos de los pisos Montehermosense y Chapadmalalense (Plioceno) del esquema cronológico sudamericano. Ameghiniana 32 (4): 369-374.

Cione, A.L.; Tonni, E.P. 2001. Correlation of Pliocene to Holocene southern South American and European vertebrate-bearing units. Bolletino della Societa Paleontologica Italiana 40 (2): 167-173.

Cione, A.L.; Tonni, E.P. 2005. Bioestratigrafía basada en mamíferos del Cenozoico superior de la provincia de Buenos Aires, Argentina. In Geología y Recursos Minerales de la Provincia de Buenos Aires (De Barrio, R.E.; Etcheverry, R.O.; Caballé, M.F.; Llambías, E.; editores). Congreso Geológico Argentino, No. 16, Relatorio 11: 183-200. La Plata.

Cione, A.; Azpelicueta, M.M.; Bond, M.; Carlini, A.; Casciotta, J.; Cozzuol, M.; De la Fuente, M.; Gasparini, Z.; Goin, F.; Noriega, J.; Scillato Yané, G.; Soibelzon, L.; Tonni, E.P.; Verzi, D.; Vucetich, G. 2000. Miocene vertebrates from Entre Ríos province, eastern Argentina. 
In El Neógeno de Argentina (Aceñolaza, F.G.; Herbst, R.; editores). Instituto Superior de Correlación Geológica (INSUGEO), Serie Correlación Geológica 14: 191-237.

Contreras, V.H.; Baraldo, J.A. 2011. Calibration of the Chasicoan-Huayquerian stages boundary (Neogene), San Juan, western Argentina. In Cenozoic Geology of the Central Andes of Argentina (Salfity, J.A.; Marquillas, R.A.; editors). Instituto del Cenozoico, Universidad Nacional de Salta: 111-121.

Del Río, C.J.; Martínez, S.A.; McArthur, J.M.; Thirlwall, M.F.; Pérez, L.M. 2018. Dating late Miocene marine incursions across Argentina and Uruguay with Srisotope stratigraphy. Journal of South American Earth Sciences 85: 312-324.

Deschamps, C.M. 2005. Late Cenozoic mammal biochronostratigraphy in southwestern Buenos Aires province, Argentina. Ameghiniana 42: 733-750.

Desmarest, A.G. 1817. Gerboise première espèce: la grande gerboise Dipus maximus Blainv. Nouveau Dictionnaire d'Histoire Naturelle 13: 117-119.

Dozo, M.T.; Monti, A.; Bouza, P.; Vucetich, M.G.; Cione, A.; Tonni, E.; Scillato-Yané, G. 2002. Geología y vertebrados continentales en cercanías de Punta Delgada (Neógeno de Península Valdés, Chubut, Argentina). In Congreso Geológico Argentino, No. 15 (Cabaleri, N.; Cingolani, C.A.; Linares, E.; López de Luchi, M.G.; Ostera, H.A.; Panarello, H.O.; editores), Actas 1: 536-541. El Calafate.

Dozo, M.T.; Bouza, P.; Monti, A.; Palazzesi, L.; Barreda, V.; Massaferro, G.; Scasso, R.A.; Tambussi, C.P. 2010. Late Miocene continental biota in Northeastern Patagonia (Península Valdés, Chubut, Argentina). Palaeogeography, Palaeoclimatology, Palaeoecology 297: 100-109.

Ercoli, M.D.; Álvarez, A.; Santamans, C.; González Patagua, S.A.; Villalba Ulberich, J.P.; Constantini, O.E. 2019. Los Alisos, a new fossiliferous locality for Guanaco Formation (late Miocene) in Jujuy (Argentina), and a first approach of its paleoecological and biochronology implications. Journal of South American Earth Sciences 93: 203-213.

Esteban, G.I.; Nasif, N.L.; Montalvo, C.I. 2001. Nuevos registros de Dasypodidae (Xenarthra) del Mioceno tardío de la provincia de La Pampa, Argentina. Revista Española de Paleontología 16: 77-87.

Esteban, G.; Nasif, N.; Georgieff, S.M. 2014. Cronobioestratigrafía del Mioceno tardío-Plioceno temprano, Puerta de Corral Quemado y Villavil, provincia de Catamarca, Argentina. Acta Geológica Lilloana 26 (2): 165-192.
Forasiepi, A.M.; Prevosti, F.J.; Vera, B.; Turazzini, G.F.; Echarri, S.; Garrido, A.C.; Verzi, D.; Rasia, L.L.; Schmidt, G.I.; Esteban, G.; Krapovikas, V. 2014. The badlands from Mendoza and the Huayquerian Age: insights into the late Miocene. In International Paleontological Congress, No. 4, Abstract volume: p. 713. Mendoza.

Forasiepi, A.M.; Prevosti, F.J.; Garrido, A.C.; Vera, B.; Turazzini, G.F.; Echarri, S.; Bonini, R.; Pujos, F.; MacPhee, R.; Verzi, D.; Rasia, L.L.; Schmidt, G.I.; Esteban, G.; Krapovikas, V. 2016. Avances en el conocimiento de la fauna de la Formación Huayquerías (Mioceno tardío, Mendoza). Ameghiniana 53 (1): 12-13R.

Francis, J.C.; Mones, A. 1965. La presencia de vizcachas [Lagostomus (Lagostomopsis) spicatus (Amegh.)] en la Formación Kiyú, Dto. de San José, R. O. del Uruguay. Revista de la Facultad de Humanidades y Ciencias 22: 155-168. Montevideo.

Francis, J.C.; Mones, A. 1968. Los roedores fósiles de Uruguay. Boletín del Laboratorio de Paleontología de Vertebrados 1 (2): 35-64.

Goin, F.J.; Montalvo, C.I.; Visconti, G. 2000. Los Marsupiales (Mammalia) del Mioceno Superior de la Formación Cerro Azul (Provincia de La Pampa, Argentina). Estudios Geológicos 56 (1-2): 101-126.

Herbst, R.; Anzótegui, L.; Esteban, G.; Mautino, L.; Morton, S.; Nasif, N. 2000. Síntesis paleontológica del Mioceno de los valles Calchaquíes del noroeste argentino. In El Neógeno de Argentina (Aceñolaza, F.; Herbst, R.; editores). Instituto Superior de Correlación Geológica (INSUGEO), Serie de Correlación Geológica 14: 263-287.

Jackson, J.E.; Branch, L.C.; Villareal, D. 1996. Lagostomus maximus. Mammalian Species 543: 1-6.

Johnson, N.M.; Jordan, T.E.; Johnsson, P.A.; Naeser, C.W. 1986. Magnetic Polarity Stratigraphy, Age and Tectonic Setting of Fluvial Sediments in an Eastern Andean Foreland Basin, San Juan Province, Argentina. In Foreland Basins (Allen, P.A.; Homewood, P.; editors). International Sedimentological Association, Special Publication 8: 63-76.

Kerber, L.; Pereira Lopes, R.; Vucetich, M.G.; Ribeiro, A.M.; Pereira, J. 2011. Chinchillidae and Dolichotinae rodents (Rodentia: Hystricognathi: Caviomorpha) from the late Pleistocene of Southern Brazil. Revista Brasileira de Paleontologia 14 (3): 229-238.

Kraglievich, L. 1926. Sobre el conducto humeral en las vizcachas y paquirucos chapadmalenses con descripción 
del Paedotherium imperforatum. Anales del Museo Nacional de Historia Natural 34: 45-51.

Kraglievich, L. 1934. La antigüedad Pliocena de las Faunas de Monte Hermoso y Chapadmalal, deducidas de su comparación con las que le precedieron y sucedieron. Imprenta El Siglo Ilustrado: 136 p. Montevideo.

Llanos, A.C.; Crespo, J.A. 1952. Ecología de la vizcacha (Lagostomus maximus maximus Blainv.) en el nordeste de la Provincia de Entre Ríos. Revista de Investigaciones Agrícolas 6: 289-378.

Lucero, N.P. 2016. Bioestratigrafía y paleontología del Neógeno y Cuaternario de San Luis, Argentina. Tesis Doctoral (Inédito), Universidad Nacional de San Luis: 202 p.

Marshall, L.G.; Patterson, B. 1981. Geology and geochronology of the mammal-bearing Tertiary of the Valle de Santa María and Río Corral Quemado, Catamarca Province, Argentina. Fieldiana Geology (New Series) 9: 1-80.

Marshall, L.G.; Sempere, T. 1991. The Eocene to Pleistocene vertebrates of Bolivia and their stratigraphic context: a review. In Fósiles y Facies de Bolivia, Volumen I-Vertebrados (Suárez-Soruco, R.; editor). Revista Técnica de Yacimientos Petrolíferos Fiscales Bolivianos (YPFB) 12 (3-4): 631-652.

Milana, J.P.; Bercowski, F.; Jordan, T. 2003. Paleoambientes y magnetoestratigrafía del Neógeno de la Sierra de Mogna, y su relación con la Cuenca de Antepaís Andina. Revista de la Asociación Geológica Argentina 58 (3): 447-473.

Montalvo, C.I.; Tomassini, R.L.; Sostillo, R.; Cerdeño, E.; Verzi, D.H.; Visconti, G.; Folguera, A.; Schmidt, G.I. 2019. A chasicoan (late Miocene) vertebrate assemblage from Cerro Azul Formation, central Argentina. Geomorphological and biochronological considerations. Journal of South American Earth Sciences 95: 102218. doi: 10.1016/j.jsames.2019. 102218.

Nasif, N.L.; Candela, A.; Rasia, L.; Madozzo Jaén, M.C.; Bonini, R. 2013. Actualización del conocimiento de los Roedores del Mioceno tardío de la Mesopotamia Argentina: aspectos sistemáticos, evolutivos y paleobiogeográficos. In El Neógeno de la Mesopotamia Argentina (Brandoni, D.; Noriega, J.; editores). Asociación Paleontológica Argentina, Publicación Especial 14: 147-163.

Nowak, R.M. 1991. Walker's Mammals of the World, $5^{\text {th }}$ Ed. The John Hopkins University Press: 1629 p. Baltimore.
Pardiñas, U.F.J.; Prevosti, F.J.; Voglino, D.; Cenizo, M. 2017. A controversial unit within the argentine neogene: the "Irenean" fauna. Ameghiniana 54: 655-680.

Pascual, R. 1966. Vertebrata. In Paleontografía Bonaerense, Volumen 1 (Borrello, A.V.; editor). Comisión de Investigaciones Científicas de la Provincia de Buenos Aires: 1-202. Buenos Aires.

Pascual, R.; Bondesio, P. 1963. Nuevo tipo de morfología dentaria de un Cardiatheriinae (Rodentia: Hydrochoridae) del Plioceno inferior de Huachipampa (San Juan). Ameghiniana 3 (2): 43-49.

Perea, D.; Rinderknecht, A.; Ubilla, M.; Bostelmann, E.; Martínez, S. 2013. Mamíferos y estratigrafía del Neógeno de Uruguay. In El Neógeno de la Mesopotamia Argentina (Brandoni, D.; Noriega, J.; editores). Asociación Paleontológica Argentina, Publicación Especial 14: 192-206.

Prado, J.L.; Chiesa, J.; Tognelli, G.; Cerdeño, E.; Strasser, E. 1998. Los mamíferos de la Formación Río Quinto (Plioceno), Provincia de San Luis (Argentina). Aspectos bioestratigráficos, zoogeográficos y paleoambientales. Estudios Geológicos 54: 153-160.

Rasia, L.L. 2016. Los Chinchillidae (Rodentia, Caviomorpha) fósiles de la República Argentina: sistemática, historia evolutiva y biogeográfica, significado bioestratigráfico y paleoambiental. Tesis Doctoral (Inédito), Universidad Nacional de La Plata, Facultad de Ciencias Naturales y Museo: $381 \mathrm{p}$.

Rasia, L.L.; Candela, A.M. 2013. Systematic and biostratigraphic significance of a chinchillid rodent from the Pliocene of eastern Argentina. Acta Palaeontologica Polonica 58 (2): 241-254.

Rasia, L.L.; Candela, A.M. 2017a. Lagostomus telenkechanum sp. nov., a new lagostomine rodent (Caviomorpha, Chinchillidae) from the Arroyo Chasicó Formation (late Miocene; Buenos Aires Province, Argentina). Journal of Vertebrate Paleontology 37 (1): e1239205. doi: $10.1080 / 02724634.2017 .1239205$.

Rasia, L.L.; Candela, A.M. 2017b. Systematic revision of the vizcachas (Rodentia, Caviomorpha, Chinchillidae) from the Chapadmalal Formation, late Pliocene of Buenos Aires Province, Argentina. Ameghiniana 54 (1): 50-69.

Rasia, L.L.; Candela, A.M. 2019. Prolagostomus amplus Ameghino is a junior synonym of the Patagonian rodent Pliolagostomus notatus Ameghino (Chinchillidae; early Miocene, Santa Cruz Formation). Ameghiniana 56 (1): 72-77.

Reguero, M.A.; Candela, A.M. 2011. Late Cenozoic mammals from the northwest of Argentina. In Cenozoic 
Geology of the Central Andes of Argentina (Salfity, J.A.; Marquillas, R.A.; editors). Instituto del Cenozoico, Universidad Nacional de Salta: 411-426.

Rovereto, C. 1914. Los estratos araucanos y sus fósiles. Anales del Museo Nacional de Historia Natural de Buenos Aires 25: 1-247.

Rowlands, I.W. 1974. Mountain viscacha. In The Biology of Hystricomorph Rodents (Rowlands, I.W.; Weir, B.J.; editors). Symposia of the Zoological Society of London 34: 131-142.

Spotorno, A.E.; Patton, J.L. 2015. Superfamily Chinchilloidea Bennet 1883. In Mammals of South America, Vol. 2, Rodents (Patton, J.L.; Pardiñas, U.F.J.; D’Elía, G.; editors). The University of Chicago Press: 762-782. Illinois.

Spotorno, A.E.; Valladares, P. 2016. Family Chinchillidae. In Handbook of the Mammals of the World: Lagomorphs and Rodents I, Vol. 6 (Wilson, D.E.; Lacher Jr., T.E.; Mittermeier, R.A.; editores). Lynx Editions: 462-481. Madrid.

Spotorno, A.E.; Zuleta, C.A.; Valladares, J.P.; Deane, A.M.; Jiménez, J.E. 2004. Chinchilla laniger. Mammalian Species 758: 1-9.

Sprechmann, P.; Ferrando, L.A.; Martínez, S. 2000. Estado actual de los conocimientos sobre la Formación Camacho (Mioceno? superior?), Uruguay. In El Neógeno de Argentina (Aceñolaza, F.G.; Herbst, R.; editores). Instituto Superior de Correlación Geológica (INSUGEO), Serie Correlación Geológica 14: 47-65.

Tauber, A.A. 2005. Mamíferos fósiles y edad de la Formación Salicas (Mioceno tardío) de la sierra de Velasco, La Rioja, Argentina. Ameghiniana 42 (2): 443-460.

Tomassini, R.L.; Montalvo, C.I.; Deschamps, C.M.; Manera, T. 2013. Biostratigraphy and biochronology of the Monte Hermoso Formation (early Pliocene) at its type locality, Buenos Aires Province, Argentina. Journal of South American Earth Sciences 48: 31-42.

Tomassini, R.L.; Montalvo, C.I.; Verzi, D.H.; Deschamps, C.M.; Garrone, M.C. 2017. Bioestratigrafía de da Formación Arroyo Chasicó (Mioceno superior) en su localidad tipo, Provincia de Buenos Aires, Argentina. Ameghiniana 54 (4): 50R.

Tullberg, T. 1899. Ueber das System der Nagethiere: eine phylogenetische Studie. Nova Acta Regiae Societatis Scientiarium Upsaliensis 3: 1-514.
Ubilla, M.; Rinderknecht, A. 2016. Lagostomus maximus (Desmarest) (Rodentia, Chinchillidae), the extant plains vizcacha in the Late Pleistocene of Uruguay. Alcheringa 40 (3): 354-365.

Valladares, P.; Spotorno, A.E.; Cortés, A.; Zuleta, C. 2018. Chinchilla chinchilla (Rodentia: Chinchillidae). Mammalian Species 50 (960): 51-58.

Verzi, D.H.; Deschamps, C.M.; Montalvo, C.I. 2004. Bioestratigrafía y biocronología del Mioceno tardío de Argentina central. Ameghiniana 41 (4): 21R.

Verzi, D.H.; Montalvo, C.I.; Deschamps, C.M. 2008. Biostratigraphy and biochronology of the Late Miocene of central Argentina: evidence from rodents and taphonomy. Geobios 41: 145-155.

Villarroel, C.; Marshall, L.G. 1989. A new fossil land mammal locality of Late Miocene (Huayquerian) age from Muyu Huasi, southcentral Bolivia. Boletín del Servicio Geológico de Bolivia, Serie A, 4 (1): 27-40. La Paz.

Visconti, G.; Melchor, R.N.; Montalvo, C.I.; Umazano, A.M.; De Elorriaga, E.E. 2010. Análisis litoestratigráfico de la Formación Cerro Azul (Mioceno Superior) en la provincia de La Pampa. Revista de la Asociación Geológica Argentina 67 (2): 257-265.

Weir, B.J. 1974. The tuco-tuco and plains viscacha. In The Biology of Hystricomorph Rodents (Rowlands, I.W.; Weir, B.J.; editors). Symposia of the Zoological Society of London 34: 113-128.

Wiegmann, A.F.A. 1835. Bericht über die Fortschritte der Zoologie im Jahre 1834. Archiv für Naturgeschichte 1: 255-348.

Wood, A.E. 1955. A revised classification of the rodents. Journal of Mammalogy 36: 165-187.

Woods, C.A.; Howland, E.B. 1979. Adaptive radiation of capromyid rodents: anatomy of the masticatory apparatus. Journal of Mammalogy 60: 95-116.

Zárate, M.A.; Schultz, P.H.; Blasi, A.; Heil, C.; King, J.; Hames, W. 2007. Geology and geochronology of type Chasicoan (late Miocene) mammal-bearing deposits of Buenos Aires (Argentina). Journal of South American Earth Sciences 23: 81-90.

Zimicz, N.; Payrola, P.; Del Papa, C. 2018. New, Late Miocene mammalian assemblage from the Palo Pintado Formation (Northwestern Argentina). Journal of South American Earth Sciences 81: $31-44$. 


\section{Apéndice}

\section{Listado del material de comparación utilizado}

Lagostomus maximus. MACN-Ma 48.274, 48.276, 48.277, 48.279, 48.280, 48.281, 49.5, 49.8, 49.12, 49.13, $49.14,49.16,49.17,49.43,49.44,49.45,49.46,49.48,49.141,49.289,49.291,50.10,50.13,50.14,50.15$, $50.17,50.18,50.20,50.21,53.8,53.9,53.10,53.11,53.13,53.19,53.20,53.21,53.24,53.25,53.27$, 53.33, 53.34, 53.35, 53.36, 53.37; MLP-Mz 14, 19, 37, 38, 39, 41, 42, 45, 54, 59, 61, 64, 230, 254, 269, $338,379,565,720,1473,1602,1603,1604,1605,1634,1642,1651,1657,1659,1728$.

Lagostomus telenkechanum. MLP-Pv 60-VI-18-101 (holotipo), MLP-Pv 55-IV-28-42, 55-IV-28-43, 55-IV28-44, 55-IV-28-44, 60-VI-18-100, 60-VI-18-107, 76-VI-12-47, 92-XI-19-1, 92-XI-19-2.

Lagostomus antiquus. MASP 32 (holotipo), CICYTTP-PV M-2-221, MLP-Pv 97-X-3-1, 15-424, MACN-Pv 9099, 13436, 13457, MACN-A 5883.

Lagostomus laminosus. MACN-A 8883 (holotipo); MACN-Pv 5884.

Lagostomus pretrichodactyla. MACN-Pv 8339 (holotipo); MACN-Pv 8337, 8345, 8212, 8272, 8274, 8294, $8295,8338,8340,8341,8342,8343,8344,8443,8462$.

Lagostomus incisus. MACN-A 1112 (holotipo), MACN-A 1654, MACN-Pv 7388, MLP-Pv 46-V-13-72, 48-XII-16-194, 63-VI-10-59, 86-VI-20-13, 88-VI-1-2, 91-III-1-9, 91-III-1-18, 91-III-1-36, 91-III-1-88, 91-IV-5-223, 91-IV-5-214, 91-IV-5-258, 94-II-1-136, 94-II-1-146, 01-I-10-30, 01-I-10-31, 01-I-10-32, 01-I-10-33, 01-I-10-34, 01-I-10-35, 01-I-10-37, 01-I-10-38, 01-I-10-41, 01-I-10-44, 01-I-10-47, 01-I10-52, 01-I-10-53, 01-I-10-54.

Lagostomus euplasius. MACN-Pv 6163 (holotipo), MACN-Pv 5986, 5985, MLP-Pv 54-X-13-1, 52-IX-2862, 52-IX-29-74, 52-X-1-13, 52-X-4-21, 52-XI-5-8, 91-IV-5-334, 01-I-10-36, 01-I-10-39, 01-I-10-40, 01-I-10-49, 01-I-10-50, 01-I-10-55, 01-I-10-56.

Lagostomus compressidens. MLP-Pv 54-X-13-2 (holotipo), MLP-Pv 54-X-13-4, MLP-Pv 90-VI-1-1.

Lagostomus debilis. MACN-A 1255 (holotipo), MLP-Pv 00-IX-6-1.

Lagostomus heterogenidens. MACN-A 1187 (holotipo).

Lagostomus minimus. MACN-A 1098 (holotipo).

Lagostomus cavifrons. MACN-A 1651 (holotipo), MACN-A 2175, MLP-Pv 52-IX-30-36, 52-X-2-1, 52-X5-9, 91-IV-30-17, 96-VIII-4-9.

Lagostomus egenus. MACN-A 417 (holotipo). 